\title{
Betätigungs... was?!
}

\author{
Fachterminologie nach Anne G. Fisher Betätigung spielt in der Ergotherapie eine zentrale \\ Rolle. Auch Anne G. Fisher unterstreicht ihre Bedeutung und verwendet in der Terminologie \\ des OTIPM die Begriffe betätigungszentriert, betätigungsfokussiert und betätigungsbasiert. \\ Doch was genau bedeuten diese Begriffe überhaupt und wie unterscheiden sie sich?
}

$\rightarrow$ Prof. Dr. Anne G. Fisher, Ergotherapeutin aus den USA und eine der weltweit renommiertesten Wissenschaftlerinnen der Ergotherapie, entwickelte das Occupational Therapy Intervention Process Model (OTIPM). Mithilfe einer klar definierten Terminologie erläutert sie darin die Kernkompetenzen der zeitgenössischen Ergotherapie. Sie beschäftigt sich insbesondere mit den Begriffen rund um die Betätigung als zentrales Element der Ergotherapie. Die Ausdrücke „betätigungszentriert“, „,betätigungsfokussiert“ und „betätigungsbasiert“ erscheinen zunächst sehr ähnlich und erwecken den Anschein, Synonyme für ein und dasselbe zu sein. Bei genauerer Betrachtung haben sie allerdings verschiedene Bedeutungen, die sich um Betätigung drehen. Um der Verwirrung entgegenzuwirken und eine einheitliche Verwendung von korrekter Fachterminologie zu fördern, werden die Begriffe im Folgenden erläutert und an einem Fallbeispiel veranschaulicht.

Betätigungszentriert $\rightarrow$ Der Begriff der Betätigungszentrierung umschreibt die berufsspezifische Perspektive, die alle Ergotherapeuten während des gesamten Therapieprozesses einnehmen [1]. Dazu gehört, dass man Menschen als „occupational beings“ (handelnde Wesen) betrachtet und die Bedeutung von Betätigungsanliegen anerkennt und berücksichtigt. Alles, was Ergotherapeuten machen, sollte auf diese Kernannahmen zurückzuführen sein [1]. Auch das Wissen, das sie aus Bezugswissenschaften wie der Psychologie oder Neurologie beziehen, sollte immer mit den Gedanken der betätigungszentrierten Therapie vernetzt sein [1].

Der Top-down-Ansatz kann Ergotherapeuten dabei unterstützen, die Betätigungen eines Menschen im Alltag in den Vordergrund zu stellen. Beim Bottom-up-Ansatz hingegen sind Körperfunktionen und Personenfaktoren vordergründig. Dieser funktionsorientierte Ansatz sollte nicht ausschließlich Anwendung finden, er kann aber eine Ergänzung zur betätigungszentrierten Therapie darstellen.

Modelle wie das Model of Human Occupation (MOHO), die Betätigung in den Vordergrund stellen, helfen dabei, einen betätigungs- zentrierten Blick auf Klienten zu erhalten [1]. Das MOHO beschreibt, wie die Elemente Volition, Habituation, Performanzkapazität und Umwelt in Wechselwirkung zueinander stehen und wie sie die Ausführung von Betätigung beeinflussen [2].

Betätigungsfokussiert $\rightarrow$ Liegt der Schwerpunkt der ergotherapeutischen Herangehensweise auf der Betätigung, spricht man von betätigungsfokussiertem Vorgehen. Praktisch bedeutet dies, dass sich Ergotherapeuten bei der Evaluation und Intervention auf die Betätigung konzentrieren, der Klient diese aber nicht direkt ausführt. Ein Beispiel für eine betätigungsfokussierte Evaluation wäre die Durchführung des Canadian Occupational Performance Measure (COPM). Hierbei erhebt ein Therapeut die Betätigungsanliegen des Klienten, es erfolgt jedoch keine Performanzanalyse der Betätigungen. Der Klient führt sie nicht direkt aus, stattdessen stehen Aktivitäten im Vordergrund, die die Betätigung in den Fokus stellen. Ein Beispiel dafür wäre im Rahmen einer Intervention nach dem Cognitive Orientation to daily Occupational Performance (CO-OP), wenn sich eine Ergotherapeutin und ein Klient gemeinsam Videos zu der gewünschten Betätigung anschauen oder im Internet nach Tipps recherchieren, wie man sie mithilfe von gewissen Hilfsmitteln ausführen kann.

Betätigungsbasiert $\rightarrow$ Der Ausdruck betätigungsbasiert bezeichnet die Betätigung als Hauptbestandteil der Ergotherapie. Das bedeutet, dass Betätigung sowohl in der Evaluation als auch in der Intervention ein grundlegender Bestandteil sein sollte [1]. Eine betätigungsbasierte Evaluation beinhaltet Analysen der Betätigungsperformanz und der Aufgaben, die zu einer erfolgreichen Ausführung der Betätigung gehören. Fisher sagt, dass eine betätigungsbasierte Intervention eine für den Klienten bedeutungsvolle Betätigung beinhaltet, die er direkt ausführt und an denen er arbeitet.

Das Assessment of Motor and Process Skills (AMPS) stellt eine Möglichkeit für eine betätigungsbasierte Evaluation dar [1]. Hierbei 


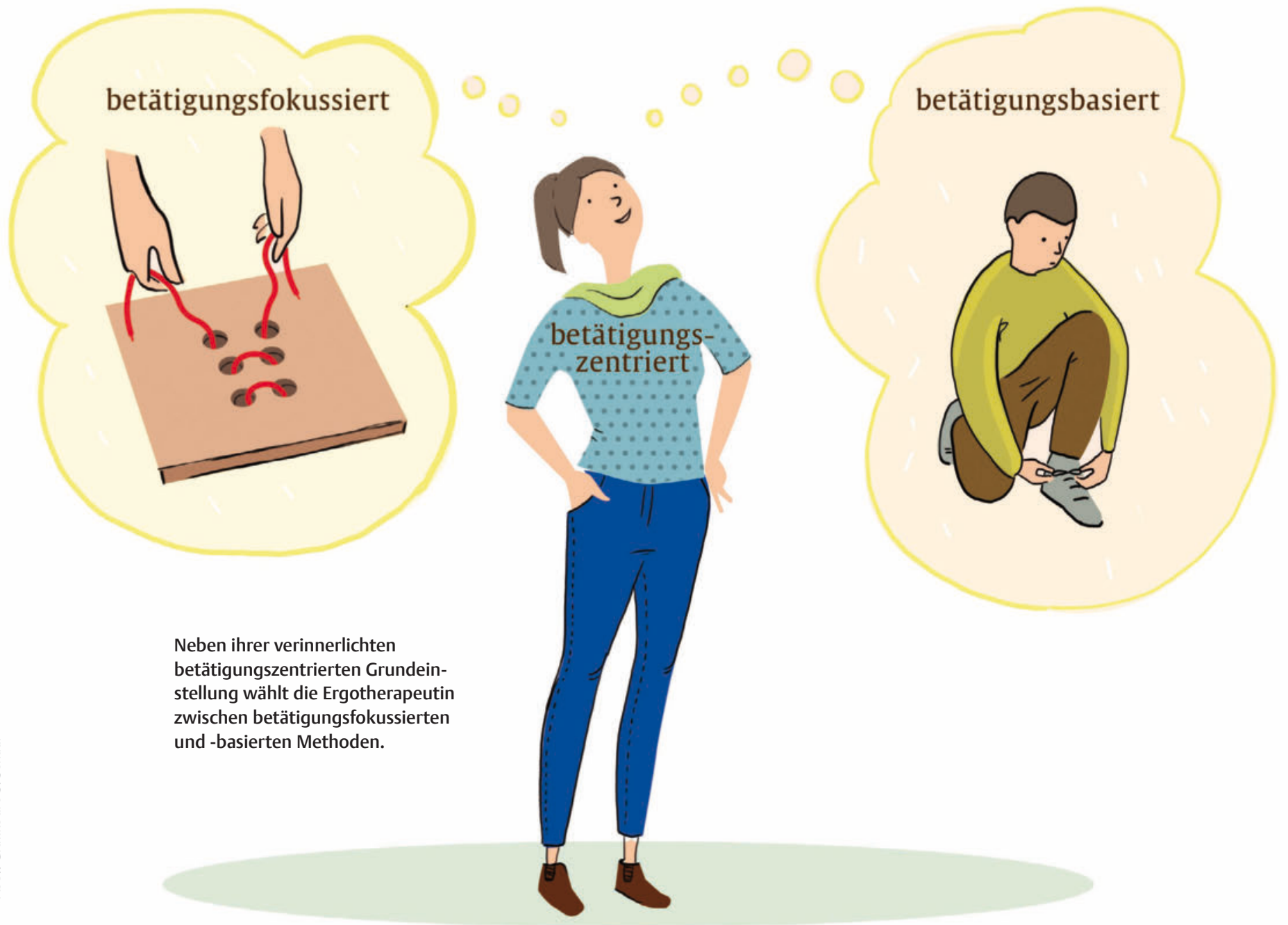

beobachtet die Therapeutin die Betätigung unmittelbar, während der Klient sie in seiner gewohnten Umgebung durchführt.

Der Einsatz von betätigungsbasierten Evaluations- und Interventionsmethoden grenzt die Ergotherapie von anderen Professionen ab. Sie verdeutlichen die einzigartige betätigungszentrierte Perspektive der Ergotherapie und setzen das Kernelement der bedeutungsvollen Betätigung mit ihnen um [1]. Wichtig ist, dass man die Betätigung so wählt, wie sie gewöhnlich im Alltag des Klienten abläuft und dass sie den Zielen des Klienten entspricht [1].

Terminologie in der Praxis $\rightarrow$ Im folgenden Fallbeispiel werden die drei Begriffe betätigungszentriert, betätigungsfokussiert und betätigungsbasiert und ihre Unterscheidung verdeutlicht. Herr Bauer* ist 55 Jahre alt und hat die Diagnose „schlaffe Hemiparese links“ nach einem Schlaganfall. Aufgrunddessen bekommt er von seinem Hausarzt eine Verordnung für Ergotherapie.

Bereits im Erstkontakt am Telefon legt die Therapeutin großen Wert darauf, Herrn Bauer über die Grundgedanken und -prinzipien der betätigungsorientierten Ergotherapie aufzuklären. Somit kann auch er von Beginn an eine betätigungszentrierte Perspektive einnehmen. Hierzu fragt sie ihn während des Telefongesprächs zunächst, ob er schon eine Vorstellung von Ergotherapie hat und wie diese aussieht. Der Klient sagt, dass er bereits in der Rehaklinik an einer Frühstücksgruppe teilgenommen habe, in der er mit der Unterstützung von Ergotherapeuten üben konnte, sich sein Frühstück wieder alleine zuzubereiten. Die Therapeutin anwortet daraufhin, dass das Zurückerlangen von Selbstständigkeit und Handlungskompetenzen einen großen Teil der Ergotherapie darstellt. Dann ergänzt sie noch, dass Herr Bauer im Sinne der Klientenzentrierung selbst entscheiden wird, an welchen Problemen aus seinem täglichen Leben er arbeiten möchte, und dass sie auf dieser Grundlage gemeinsam für ihn bedeutsame Ziele für die Therapie entwickeln werden.

Als der 55-jährige Klient zum Erstgespräch in die Ergotherapiepraxis kommt, führt die Therapeutin mit ihm das COPM durch, um einen Einblick in seinen Alltag zu bekommen. Sie sprechen über seine täglichen Routinen, Betätigungsherausforderungen und Betä- 


\section{Ergotherapie | Fachterminologie}

tigungsanliegen. Das Erstgespräch ist somit - im Sinne der Terminologie nach Fisher - betätigungsfokussiert ausgerichtet, denn Herrn Bauers Betätigungen sind zwar das zentrale Thema des Gesprächs, er führt sie jedoch nicht aktiv aus.

Betätigungszentrierte Perspektive $\rightarrow$ Das COPM-Interview ergibt, dass Herr Bauer vor seinem Schlaganfall ein gutes Einkommen hatte und nun mit seiner Ehefrau gemeinsam beschloss, den Lebensunterhalt der Familie zukünftig von ihrem Gehalt als freie Juristin und seiner Erwerbsminderungsrente zu bestreiten. Anstelle des Arbeitens ist es ihm nun wichtiger, sich wieder selbstständig versorgen zu können. Seine Frau unterstützt ihn momentan vor allem beim Anziehen, der Pflege und der Zubereitung von Mahlzeiten. Sie könnte ihre Termine zwar so legen, dass sie immer vor Ort ist, um ihn zu unterstützen. Dies ist aber nicht das Leben, das er sich für sich und seine Frau auf Dauer wünscht. Er ist es gewohnt, ein eigenständiger Mensch zu sein, der für sich und seine Familie sorgen kann. Hinzu kommt, dass seine Tochter einen 2-jährigen Sohn hat, mit dem er und seine Frau an den Wochenenden oft etwas unternehmen und auf den sie aufpassen. Es fehlt ihm sehr, seinen Enkel zu tragen und mit ihm zu spielen. Der 55-Jährige hat außerdem einen aktiven Freundeskreis, mit dem er sich regelmäßig trifft. Für längere Strecken muss er sich im Rollstuhl schieben lassen, was ihn sehr stört. Im Haus selbst kann er sich aber - dank Gehstock und vieler Haltegriffe - ohne Hilfsperson fortbewegen.

Diese Informationen aus dem Erstgespräch sortiert die Ergotherapeutin in die Elemente des MOHO-Modells ein. Das unterstützt sie dabei, eine betätigungszentrierte Perspektive auf die Situation und die Probleme des Klienten zu bekommen. Die Betätigungsanliegen, die durch das COPM ermittelt wurden, nutzt die Therapeutin als Argumentationsgrundlage, um beim behandelnden Hausarzt eine Änderung der Verordnung auf Hausbesuche zu erwirken, damit die Therapie im gewohnten Setting stattfinden kann.

\section{פ5}

\section{Betätigungsbasierte Interventions- methoden grenzen die Ergotherapie von anderen Berufsgruppen $a b$.}

Betätigungsanalyse im Wohnumfeld $\rightarrow$ Für die darauffolgende Therapieeinheit vereinbart die Ergotherapeutin mit Herrn Bauer, das morgendliche Waschen und Anziehen zu analysieren. Sie einigen sich auf einen Termin, bei dem auch die Ehefrau anwesend sein kann. Die Therapeutin sieht sich die Betätigung im gewohnten Setting an, ohne eine Verfälschung der Umstände. Die Performanz, die sich während der Betätigung beobachten lässt, beurteilt sie mit dem AMPS. Folglich ist die Evaluation betätigungsbasiert, da sie durch eine Beobachtung der Betätigung in der natürlichen Umwelt, zur gewohnten Tageszeit und unter dem Beisein von Frau Bauer erfolgt.

Alle Schritte der morgendlichen Selbstversorgung, an denen Frau Bauer ihren Mann momentan unterstützt, notiert sich die The- rapeutin. Anschließend überlegt sie auf Grundlage dessen gemeinsam mit Herrn Bauer, welche Handlungsalternativen es gibt, mit denen er die Betätigungen selbstständig bewältigen könnte. Das reflektierende Gespräch, das die Durchführung des Anziehens und Waschens zum Inhalt hat, ist im Gegensatz zur eigentlichen Durchführung betätigungsfokussiert. Nach einigen Therapieeinheiten reevaluiert die Therapeutin die Betätigung mit Herrn Bauer. Dafür

$$
\begin{gathered}
\text { Um eine Profession zu } \\
\text { positionieren, ist eine einheitliche } \\
\text { Fachsprache unabdingbar. }
\end{gathered}
$$

setzt sie wieder die betätigungsbasierte Methode des AMPS ein. Zudem ergänzt sie dies durch eine betätigungsfokussierte Methode, da sie auch das COPM erneut mit dem Klienten durchführt, um Veränderungen der Performanz und der Zufriedenheit festzustellen.

Klare Fachsprache vonnöten $\rightarrow$ Es wird deutlich, dass die Begriffe betätigungszentriert, betätigungsfokussiert und betätigungsbasiert unterschiedliche Bedeutungen für den ergotherapeutischen Prozess haben. Im aktuellen Professionalisierungsprozess im Sinne der voranschreitenden Akademisierung sowie im Hinblick auf zukünftige innovative Arbeitsfelder und die Verinnerlichung einer einheitlichen Berufsidentität ist eine klare Fachsprache erforderlich. Da die Fachterminologie noch dabei ist, sich bei allen Berufsangehörigen zu etablieren, ist das genaue Beschreiben von Begriffen unabdingbar [3]. Nur ein korrektes Verständnis und eine einheitliche Verwendung der Fachsprache ermöglichen es, sich als Berufsgruppe zu positionieren und abzugrenzen, um die wachsenden Herausforderungen und Möglichkeiten der Profession wahrnehmen zu können. Daniela Berg, Jamie-Lee Oster, Carolin Takacs

$\Rightarrow$ Literaturverzeichnis www.thieme-connect.de/products/ergopraxis > „Ausgabe 5/19“

\section{(6) Autorinnen}

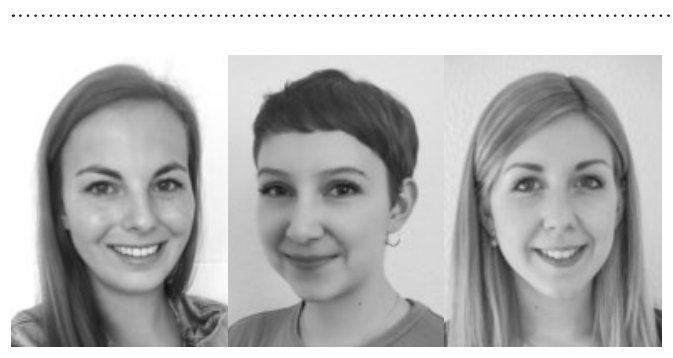

Daniela Berg, Jamie-Lee Oster und Carolin Takacs sind Studierende im zweiten Semester des Bachelorstudiengangs Ergotherapie an der Zuyd Hogeschool in Heerlen (NL). Im Rahmen einer Hausarbeit haben sie sich mit der Terminologie nach Anne G. Fisher auseinandergesetzt und einen Beitrag für ergopraxis ausgearbeitet. 